\title{
Quantificação de Resíduos Produzidos nas Indústrias Madeireiras de Gurupi, TO
}

\author{
Cândida Pereira da Silva ${ }^{1}$, Renato da Silva Vieira ${ }^{2}$, Iolanda Carvalho Silva ${ }^{1}$, \\ Aline Silvestre Pereira Dornelas ${ }^{1}$, Edy Eime Pereira Baraúna ${ }^{3}$
}

\author{
${ }^{1}$ Universidade Federal do Tocantins - UFT, Gurupi/TO, Brasil \\ ${ }^{2}$ Departamento de Ciências Agrárias, Universidade Federal de São João Del Rei - UFSJ, Sete Lagoas/MG, Brasil \\ ${ }^{3}$ Instituto de Ciências Agrárias - ICA, Universidade Federal de Minas Gerais - UFMG, Montes Claros/ MG, Brasil
}

\begin{abstract}
RESUMO
O estudo foi desenvolvido no município de Gurupi, Estado do Tocantins, com o objetivo de quantificar a produção de resíduos gerados por indústrias do setor madeireiro. Foram selecionadas empresas do setor moveleiro de grande, médio e pequeno porte, além de manufaturadoras de esquadrias. O porte das empresas foi determinado pelo número de funcionários. Foram realizadas medições diretas de comprimento, largura e espessura das peças, sendo consideradas aparas, fragmentos menores que $10 \mathrm{~cm}$ de largura, e recortes, fragmentos maiores que $10 \mathrm{~cm}$ de largura, $\mathrm{e}$ a serragem e pó de serra foram medidos em caixa de volume conhecido. Em um mês de produção, a fábrica de esquadrias produziu um total de $32,23 \mathrm{~m}^{3}$ de resíduos, já a empresa de grande porte produziu $15,44 \mathrm{~m}^{3}$, a empresa de médio porte, $10,95 \mathrm{~m}^{3}$ e a empresa pequena, $8,68 \mathrm{~m}^{3}$.
\end{abstract}

Palavras-chave: setor madeireiro, industrialização, resíduo.

\section{Quantification of Waste Produced in the Gurupi, TO Lumber Industry}

\begin{abstract}
The study was conducted in the city of Gurupi, Tocantins State, in order to quantify the waste generated by industries in the lumber sector. Large, medium and small companies of the furniture and frames industry of were selected. The company size was determined by the number of employees from each company. Direct measurements of the length, width and thickness of the pieces were performed. Chips, fragments smaller than $10 \mathrm{~cm}$ wide, and cutouts fragments larger than $10 \mathrm{~cm}$ width were considered for measurements and sawdust was measured with aid of a box of known volume. Within one month, the company fabricating frames produced a total of $32.23 \mathrm{~m}^{3}$ of waste, while the large company produced $15.44 \mathrm{~m}^{3}$, the medium-size company produced $10.95 \mathrm{~m}^{3}$, and the small company produced $8.68 \mathrm{~m}^{3}$ of waste.
\end{abstract}

Keywords: timber sector, industrialization, residues.

\section{INTRODUÇÃO}

A madeira foi um dos primeiros materiais a serem utilizados pela humanidade, mantendo, apesar do aparecimento dos materiais sintéticos, uma imensidade de usos, que vão desde a confecção de utensílios domésticos a complexas estruturas prediais. A evolução da utilização da madeira é evidente, prova disto são as ferramentas utilizadas na Idade Média para a produção de armas e as modernas máquinas de usinagem utilizadas na fabricação de móveis nos dias atuais.

Devido ao grande avanço das técnicas de aplicação da madeira, esta passou a ser matéria-prima versátil, sendo base para uma grande variedade de produtos, 
tais como: celulose, papel, energia, taninos, resinas, açúcares, madeira serrada, madeira roliça e chapas de madeira reconstituída a base de fibras, partículas e lâminas (Fagundes, 2003).

O processo de industrialização da madeira acarreta a geração de resíduos, que necessitam de tratamento adequado. Apesar de a ciclagem dos resíduos ocorrer no ambiente com o passar do tempo, a deposição imprópria desse material podem trazer prejuízos ambientais significativos. Dentre estes prejuízos está a emissão de gás carbônico na atmosfera em decorrência de queima a céu aberto. Para minimizar os problemas causados pelo despejo inadequado desses resíduos, podem-se utilizar alternativas racionais como cama de forração em aviários, revestimento de jardins e vasos de planta, geração de energia, compostagem e artesanato (Mady, 2000; Fagundes, 2003).

Pesquisas apontam que os melhores índices de aproveitamento de toras de madeira serrada situam-se em torno de $35 \%$ a $55 \%$, o que justifica o estudo de possibilidades de aproveitamento desses resíduos em outros produtos ou processos (Fagundes, 2003). De acordo com Gomes \& Sampaio (2004), uso de tecnologia sofisticada, aliada ao treinamento de operários, minimiza a possibilidade de perdas e pode aumentar a eficiência no processamento da madeira, obtendo-se maior produção e menor geração de resíduos.

De acordo com Lima \& Silva (2005), os resíduos de madeira gerados por seu processamento podem deixar de ser risco ao meio ambiente e passar a gerar lucro para a empresa que os produz, além do que pode-se apresentar alternativas para sua utilização como matéria-prima de diversos outros produtos.

$\mathrm{O}$ aproveitamento de resíduos de madeira tem grande importância, pois contribui para a racionalização dos recursos florestais. A reutilização proporciona alternativas socioeconômicas às empresas que gerenciam seus resíduos de forma apropriada. O processamento de madeira pode ser incluído no rol dos geradores de resíduos que podem se transformar em poluentes ambientais caso não sejam aproveitados ou reciclados de forma adequada.

Segundo Abreu \& Azevedo (2009), fazer um diagnóstico sobre os resíduos da indústria madeireira não é um trabalho fácil, pois as características dos resíduos gerados em uma indústria são muito diversificadas, já que dependem do tipo de produto que está sendo confeccionado em um determinado período.

No município de Gurupi, TO, assim como em outras cidades de mesmo porte, encontram-se muitos indústrias que usam a madeira como fonte de matéria-prima. Os produtos de madeira comercializados por essas empresas apresentam grande variedade, desde móveis a madeira para construção. Desse modo, há produção de resíduos que podem ser aproveitados de forma mais eficiente.

O presente trabalho teve como principal objetivo quantificar a produção de resíduos gerados por indústrias do setor madeireiro no município de Guripi, TO.

\section{MATERIAL E MÉTODOS}

\subsection{Localização da área de estudo}

O projeto foi desenvolvido em fábricas de móveis, marcenarias e fábrica de esquadrias, totalizando quatro empresas (Figura 1) do município de Gurupi, região sul do Estado do Tocantins, juntamente com o apoio da Universidade Federal do Tocantins, campus de Gurupi.

\subsection{Levantamentos das empresas geradoras de resíduos sólidos de madeira}

Foram selecionadas empresas do setor moveleiro, uma de grande, uma de médio e uma de pequeno porte, além da fábrica de esquadrias. $\mathrm{O}$ porte das empresas foi definido de acordo com o número de funcionários, a empresa denominada de grande porte possui acima de 10 funcionários efetivos, na de médio porte trabalham de 3 até 10 funcionários e na empresa pequena, apenas até 3 funcionários mais o proprietário. A fábrica de esquadrias também foi considerada de grande porte, pois possui 15 funcionários, embora tenha sido classificada como categoria a parte. A coleta dos dados foi realizada durante quatro meses, de julho a outubro, 2012. As medições foram feitas semanalmente, do resíduo também produzido e estocado durante a semana pelos proprietários.

\subsection{Identificação e quantificação dos resíduos}

A identificação dos resíduos foi obtida com base no tamanho e forma dos resíduos sólidos. A classificação foi de acordo com Fontes (1994) e IBDF (1988), com adaptações: aparas foram desmembradas de recortes 


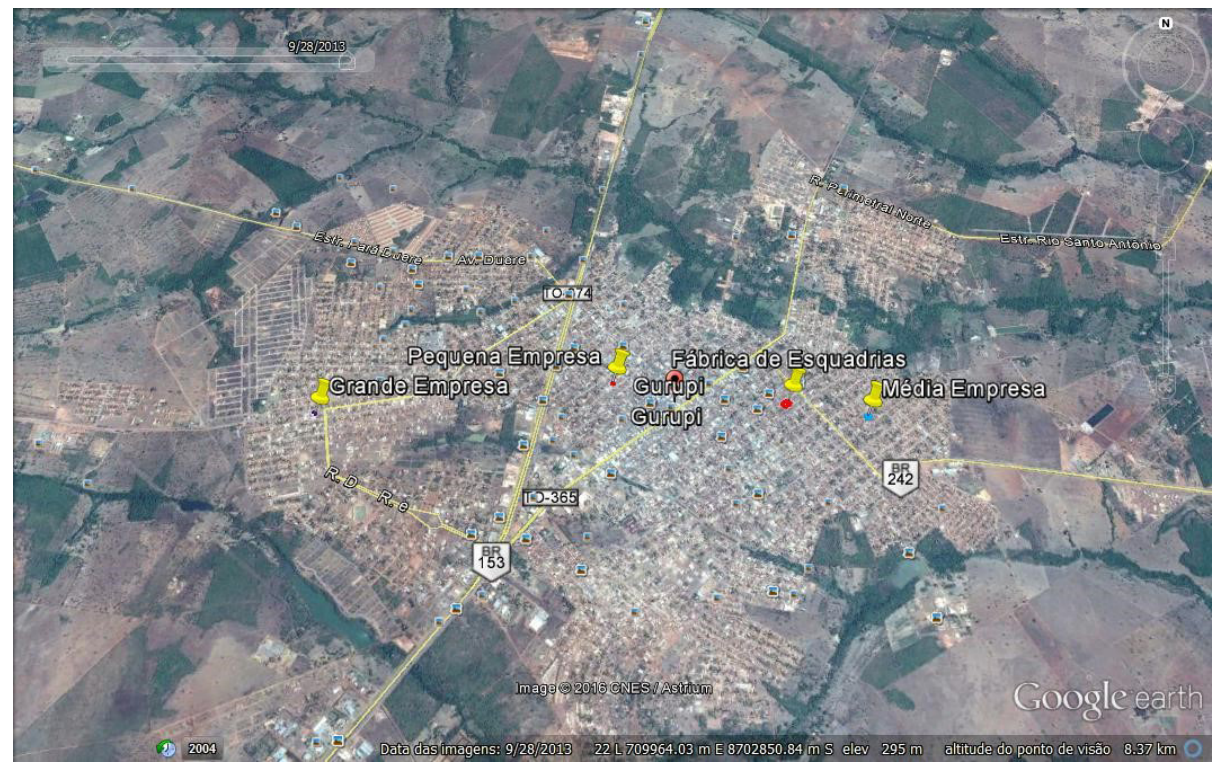

Figura 1. Localização das empresas em Gurupi, TO.

Figure 1. Location of companies in Gurupi, TO.

em função do tamanho do material de pesquisa. Aparas foram identificadas como peças menores que $10 \mathrm{~cm}$ de largura, recortes, como peças maiores que $10 \mathrm{~cm}$ de largura, serragem como madeira particulada (maravalha) e pó de serra como madeira em pó.

O cálculo do volume de recortes e aparas foi feito utilizando-se fórmulas de acordo com a figura geométrica, as mais comuns foram retângulos, quadrados e triângulos, e o volume da serragem e do pó de serra, pelo volume conhecido do recipiente de medição.

Para a medição de aparas e recortes foi utilizada medição direta com fita métrica para a obtenção das dimensões (largura, comprimento e altura). Serragem e pó de serra foram medidos em uma caixa de madeira com dimensões conhecidas: $0,60 \mathrm{~m}$ de comprimento, $0,50 \mathrm{~m}$ de largura e $0,50 \mathrm{~m}$ de altura. A tabulação dos dados foi realizada no Microsoft Excel $^{\circledR} 2007$, no qual tabelas e gráficos dos dados obtidos na pesquisa foram avaliados. Os dados foram discutidos com base na estatística descritiva.

\section{RESULTADOS E DISCUSSÃO}

Das empresas do setor moveleiro, as de grande e médio porte produziam móveis sob encomenda, de alta qualidade, que eram comercializados em lojas ligadas às movelarias. Enquanto que a de pequeno porte só trabalhava com móveis sob encomenda. A fábrica de esquadrias foi estudada como uma categoria a parte, devido sua importância no município de Gurupi, tendo em vista o crescimento da indústria da construção civil, o que acarretava uma maior demanda de madeira, principalmente para ripas, caibros, portais, portas e janelas de madeira (Silas, 2010). A análise de variância pode ser observada nas Tabelas 1, 2 e 3.

Na Tabela 1 observa-se que a interação entre a empresa e o tipo de resíduo apresentou diferença significativa ao nível de 5\% de probabilidade, ou seja, a empresa influencia no tipo de resíduo produzido. Na Tabela 2 é possível verificar que tanto a serragem como o pó de serra são os tipos de resíduos que apresentaram volumes mais significativos, ou seja, foram os resíduos que apresentaram maior volume, diferenciando-se em seu quantitativo. Embora aparas e recortes também tenham apresentado importância como resíduos sólidos de madeira, provavelmente pelo baixo volume detectado nas empresas, não apresentou diferença entre elas.

Na Tabela 3 pode se observar no resumo da análise de variância do desdobramento que as empresas apresentaram diferença significativa quando fixados os resíduos. Essa diferença pode estar ligada ao volume 
Tabela 1. Resultado da análise de variância (ANOVA) considerando as empresas, tipos de resíduo e semanas avaliadas.

Table 1. Result of analysis of variance (ANOVA), considering companies, types of waste and weeks evaluated.

\section{FV}

Empresa
Tipo de resíduo
Semanas
Empresa ${ }^{\star}$ Tipo
Empresa ${ }^{\star}$ Semanas
Tipo ${ }^{\star}$ Semanas
Erro

\section{GL}

3
3
3
9
9
9
27
3,24
8292

3
3
3
9
9
9
27
3,24
8292

3
3
3
9
9
9
27
43,24
8292

3
3
3
9
9
9
27
3,24
8292

3
3
3
9
9
9
27
43,24
8292

3
3
3
9
9
9
27
3,24
8292

3
3
3
9
9
9
27
3,24
8292

3
3
3
9
9
9
27
3,24
8292

CV(\%)

0,8292

\section{QM}

2, 4687

15,1567

0,1188

1,0792

0,2002

0,1457

0,1285

\section{FC}

$19,202^{*}$

$102,335^{*}$

$0,925 \mathrm{NS}$

$8,395^{*}$

1, 557 NS

$1,133 \mathrm{NS}$

Média geral

*Significativo; NS: não significativo; FV: fonte de variação; GL: grau de liberdade; QM: quadrado médio; FC: F calculado.

Tabela 2. Resumo da análise de variância fixando- a empresa e variando os tipos de resíduo.

Table 2. Summary of analysis of variance setting up the company and varying types of waste.

\section{FV}

Empresa/Aparas

Empresa/Serragem

Empresa/Recortes

Empresa/Pó de serra

Erro

\section{GL}

3

3

3

9

27

\section{QM}

0,0757

4, 9771

0,0120

0,6415

0,1285
FC

$0,5900 \mathrm{NS}$

$38,713^{*}$

$0,0940 \mathrm{NS}$

$4,990^{*}$

*Significativo; NS: não significativo; FV: fonte de variação; GL: grau de liberdade; QM: quadrado médio; FC: F calculado.

Tabela 3. Resumo da análise de variância da interação tipo de resíduo e empresa.

Table 3. Summary of analysis of variance of interaction residue type and company.

\begin{tabular}{lccc}
\multicolumn{1}{c}{ FV } & GL & QM & FC \\
\hline Tipo de Resíduo/Grande & 3 & 1,7095 & $13,297^{*}$ \\
Tipo de Resíduo/Média & 3 & 2,5626 & $19,932^{*}$ \\
Tipo de Resíduo/ Esquadrias & 3 & 10,2383 & $79,635^{*}$ \\
Tipo de Resíduo/Pequena & 3 & 1,8840 & $14,654^{*}$ \\
Erro & 27 & 0,1285 & \\
\hline
\end{tabular}

*Significativo; NS: não significativo; FV: fonte de variação; GL: grau de liberdade; QM: quadrado médio; FC: F calculado.

diferenciado de resíduos que cada empresa produz, o qual pode estar relacionado ao tipo de maquinário, à mão de obra utilizada e à organização da empresa como um todo.

O teste de Tukey foi também realizado ao nível de $5 \%$ de probabilidade para ranquear as empresas e os resíduos estudados, o que pode ser observados nas Tabelas 4 e 5 .

No teste de média aplicado pôde-se observar que na Tabela 4 não houve diferença entre as médias dos volumes de resíduos avaliados na pequena e na média empresa, na grande empresa e na madeireira as aparas, e os recortes não apresentaram diferença nas médias do volume de resíduo, no entanto serragem e pó de serra obtiveram diferença significativa em ralação aos demais resíduos, embora entre eles não tenha ocorrido essa diferença.

$\mathrm{Na}$ Tabela 5 observa-se que aparas, recortes e pó de serra, não apresentaram diferença nas médias de acordo com o teste de Tukey ao nível de 5\% de probabilidade, no entanto a serragem apresentou diferença entre as médias, e a empresa de grande porte obteve média menor que as demais. Isso pode ser explicado pelo maior nível de tecnologia aplicado aos equipamentos, o que resulta em uma menor produção de resíduos, serragem, em relação às outras empresas, que apresentaram um nível tecnológico menor. Essas diferenças apresentadas pelos testes de médias está ilustrada na Figura 2. Na Figura 2 
Tabela 4. Teste de média da interação empresa e tipo de resíduo.

Table 4. Test medium of interaction company and type residue.

\begin{tabular}{|c|c|c|c|c|}
\hline Tipo de empresa & Aparas & Recortes & Serragem & Pó de serra \\
\hline Pequena & 0,0196 al & 0,0028 al & 1,1375 a 1 & 0,7000 a1 \\
\hline Média & 0,0949 a 1 & 0,0428 a 1 & 1,4500 a1 a2 & 0,8500 a1 a2 \\
\hline Grande & 0,1549 al & 0,0815 al & $1,7500 \mathbf{a} 2$ & 1,3425 a2 a3 \\
\hline Fábrica de esquadrias & 0,3419 al & 0,1313 al & 3,6200 a3 & 1,5475 a3 \\
\hline
\end{tabular}

Médias seguidas pela mesma letra e o mesmo número, na coluna, não diferem entre si pelo teste de Tukey ao nível de $5 \%$ de probabilidade.

Tabela 5. Teste de média da interação tipo de resíduo e empresa de porte grande, médio e pequeno.

Table 5. Test medium of interaction type of waste and great company, type of waste and medium company, type of waste and small company, type of waste and timber.

\begin{tabular}{|c|c|c|c|c|}
\hline Tipo de resíduo & Grande & Média & Pequena & Fábrica de esquadrias \\
\hline Recortes & 0,0815 al & 0,0428 a 1 & 0,0028 al & 0,1313 al \\
\hline Aparas & 0,1548 al & 0,0949 al & 0,0196 a 1 & 0,3419 al \\
\hline Pó de serra & $1,1375 \mathbf{a} 2$ & 0,8500 a2 & $0,7000 \mathbf{a} 2$ & 1,5475 a2 \\
\hline Serragem & $1,3425 \mathbf{a} 2$ & $1,7500 \mathbf{a} 3$ & 1,4500 a3 & $3,6200 \mathbf{a} 3$ \\
\hline
\end{tabular}

Médias seguidas pela mesma letra e o mesmo número, na coluna, não diferem entre si pelo teste de Tukey ao nível de $5 \%$ de probabilidade.

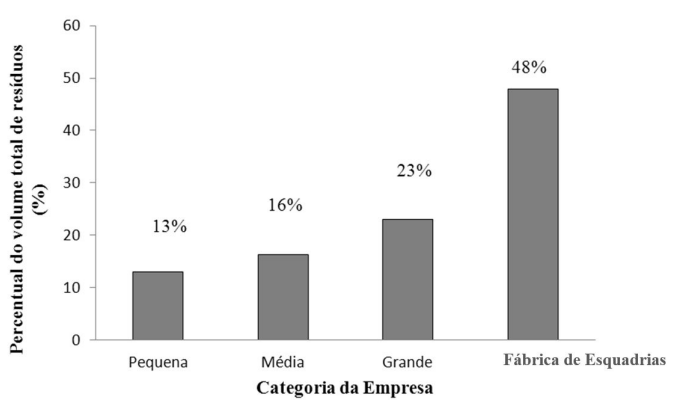

Figura 2. Gráfico do percentual do volume total (\%) de resíduos de madeira produzido por empresa de porte pequeno, médio e grande e pela fábrica de esquadrias.

Figure 2. Graph of the percentage of total volume (\%) of wood waste produced by small, medium and large company size timber.

observa-se que a diferença na produção de resíduos das indústrias é proporcional ao porte das empresas.

De acordo com os dados exibidos, pode-se observar que houve maior geração de resíduos na fábrica de esquadrias, com $48 \%$ do total quantificado em um mês, comparativamente às outras empresas estudadas, o que correspondeu a 32,23 m3 de resíduos de madeira (Tabela 6). Esse fato pode ser justificado pela maior demanda de madeira, em decorrência do aumento das atividades na construção civil no município nos últimos anos, conforme descrito por Silas (2010).
Além disso, a atividade desenvolvida pela madeireira se resume no processamento secundário da madeira, no qual os produtos acabados são, na sua maioria, peças retangulares como caibros, ripas e vigas, o que resulta em uma produtividade alta, gerando maior quantidade de resíduos que nas demais empresas estudadas.

A Figura 3 apresenta o volume total por tipo de resíduo das empresas avaliadas. Serragem e pó de serra obtiveram maior percentual em todas as empresas, com valores de $63 \%$ e $31 \%$ respectivamente. Esse resultado se deve ao fato de as empresas, pequena, média e grande, trabalharem com produção de móveis, o que resulta em uma maior geração de resíduos, devido à quantidade de etapas de processamento a que a madeira é submetida, da madeira pré-preparada ao acabamento final do móvel. Ainda deve ser considerado o rendimento da madeira em móveis, que segundo Vieira (2003) e Lima et al. (2004), é em torno de 6\%. Isso significa que de um total de $1 \mathrm{~m}^{3}$ de madeira $0,06 \mathrm{~m}^{3}$ se transformam em móveis e o restante é descartado como resíduo. De acordo com Kozak et al. (2008), a fabricação de móveis é uma prática que sempre esteve relacionada à geração de resíduos sólidos, especialmente nas etapas de beneficiamento da madeira, o que explica a grande geração de pó de serra e serragem. Porém, na fábrica de esquadrias - que também aparece como grande geradora, como mostra a Figura 2 - esses resíduos decorriam da 
maior produtividade e do tipo de maquinário utilizado nas atividades, comparativamente às outras empresas.

Observa-se na Tabela 6 que os resíduos aparas apresentou volume maior que recortes em todas as categorias de empresa, isso porque na pequena e média empresa a maioria dos fragmentos de madeira com largura acima de $10 \mathrm{~cm}$ são reaproveitados em outros produtos, como puxadores de móveis e peças decorativas, entre outros. Essa técnica é aplicada em outras empresas e apresenta viabilidade, como pode ser comprovado em trabalhos nos quais foram utilizados resíduos na confecção de kits pedagógicos e de pequenos objetos de madeira (Vieira, 2006; Vieira et al., 2010).

No entanto, diversas alternativas podem ser colocadas em prática para minimizar a poluição ambiental decorrente da incineração desses resíduos em locais inapropriados, como o seu aproveitamento pela própria empresa ou sua comercialização para outras empresas, podendo ser utilizado em cama de forração de aviários, compostagem para agricultura da região, produção de briquetes para geração de energia, dentre

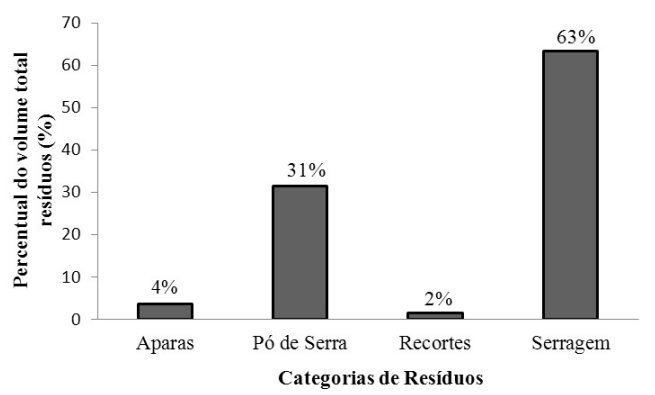

Figura 3. Gráfico do percentual do volume total das categorias de resíduo de madeira.

Figure 3. Graph oh the percentage of the total volume of the categories of waste wood. outros, aumentando a geração de renda das empresas e, consequentemente, do município de Gurupi. Apesar de a principal atividade do município estar direcionada para a pecuária, na região existem vários assentamentos rurais, os quais poderiam receber parte desses resíduos para aplicação em algumas atividades da agricultura familiar, na criação de aves e porcos.

Essas ações podem ser destacadas em diversos trabalhos, como os de Vieira et al. (2010), em que resíduos de madeira resultantes do desdobro de toras são utilizados para a produção de pequenos objetos de madeira, e de Lima et al. (2007), os quais relata alguns trabalhos em que há aproveitamento de resíduos gerados no processamento da madeira de eucalipto. No entanto, apesar de o município possuir mercado que poderia absorver todo esse resíduo, apenas a fábrica de esquadrias os aproveita, vendendo os recortes e aparas para pizzarias e padarias da cidade e a serragem e pó de serra, para agricultores da região, os quais os utilizam como cobertura morta no cultivo de melancia e mandioca, as demais empresas os descartam em terrenos baldios e depois os queimam.

Nas grandes empresas e fábricas de esquadrias, as máquinas utilizadas possuem tecnologia mais avançada e as peças são cortadas de forma predeterminada, de acordo com o que será confeccionado, o que justifica a menor geração de resíduos com largura maior que $10 \mathrm{~cm}$, classificadas neste trabalho como recortes. Não só a tecnologia do maquinário liga-se à baixa geração de resíduos: também a formação do pessoal que participa da confecção das peças que resultarão nos móveis e peças para construção. $\mathrm{O}$ treinamento de funcionários é ferramenta importante contra o desperdício (Gomes \& Sampaio, 2004).

Segundo Abreu et al. (2009), resíduos curtos ou estreitos não deixam de ser viáveis e podem ser

Tabela 6. Volume de resíduo mensal de cada categoria, gerado por diferentes tipos de empresa.

Table 6. Monthly volume of waste in each category, generated by different types of company.

\begin{tabular}{|c|c|c|c|c|}
\hline \multirow{2}{*}{$\begin{array}{l}\text { Categoria de } \\
\text { resíduo }\end{array}$} & \multicolumn{4}{|c|}{ Empresa } \\
\hline & $\begin{array}{c}\text { Pequena } \\
\text { V }\left(\mathbf{m}^{3}\right)\end{array}$ & $\begin{array}{l}\text { Média } \\
\text { V }\left(\mathbf{m}^{3}\right)\end{array}$ & $\begin{array}{l}\text { Grande } \\
\text { V }\left(\mathbf{m}^{3}\right)\end{array}$ & $\begin{array}{l}\text { Fábrica de esquadrias } \\
\qquad V\left(\mathbf{m}^{3}\right)\end{array}$ \\
\hline Aparas & 0,0784 & 0,3798 & 0,6192 & 1,3677 \\
\hline Recortes & 0,0113 & 0,1714 & 0,3262 & 0,5254 \\
\hline Pó de serra & 2,8000 & 3,4000 & 5,3000 & 9,6535 \\
\hline Serragem & 5,8000 & 7,0000 & 9,2000 & 20,6860 \\
\hline Total & 8,6897 & 10,9512 & 15,4454 & 32,2326 \\
\hline
\end{tabular}


aproveitados na confecção de pequenos objetos ou, ao menos, na de algumas partes de objetos. Porém, para que as aparas sejam utilizadas, é necessário que sejam separadas dos recortes, deve-se realizar uma seleção prévia por classe de dimensão, direcionando as peças separadas para os artesões interessados. Aquelas peças que não são possíveis de serem aproveitadas podem ser utilizadas na geração de energia, como lenha em fornos de padarias e pizzarias.

Analisando-se o volume de resíduos por semana, como mostra a Figura 4, constatou-se que estatisticamente não houve variação na quantidade de resíduos produzidos no decorrer do mês. Na segunda semana houve maior geração de resíduos, total de $15,21 \mathrm{~m}^{3}$, enquanto que na primeira semana foram gerados $13,94 \mathrm{~m}^{3}$, na terceira e quarta semanas foram gerados $13,89 \mathrm{~m}^{3}$ e $14,41 \mathrm{~m}^{3}$, respectivamente. Isso se deve ao aumento de encomendas na primeira semana do mês, possivelmente em virtude do aumento de circulação monetária no município, resultado dos salários recebidos nos primeiros dez dias úteis de cada mês.

De acordo com Silva (1999), um fator que afeta a quase totalidade das indústrias madeireiras é a flutuação da produção em razão da variação do volume comercializado ao longo do ano. Desse modo, os resultados alcançados em volume e características dos resíduos da empresa, em determinado período de tempo, não podem ser considerados para empresas de mesmo porte em localidades diferentes. Isso porque existem particularidades regionais e de sazonalidade que devem ser consideradas.

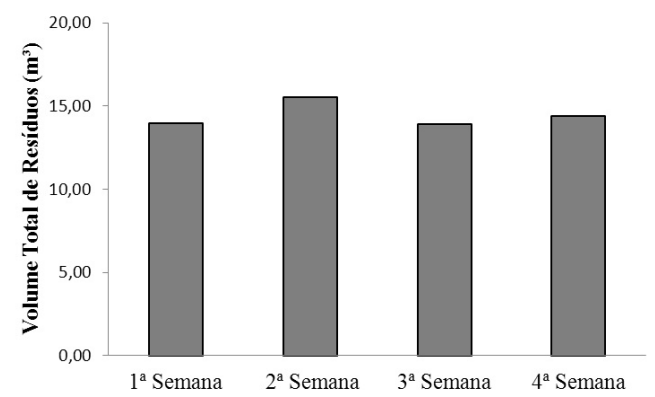

Figura 4. Volume total de resíduos produzidos nas diferentes semanas do mês.

Figure 4. Total volume of waste produced in different weeks of the month.

\section{CONCLUSÕES}

Os principais resíduos produzidos pelas empresas no município de Gurupi são aparas, recortes, serragem e pó de serra; a fábrica de esquadrias é a maior geradora de resíduos em Gurupi; serragem e pó de serra foram as categorias de resíduo que registraram maior volume dentre as estudadas; em todas as empresas houve maior geração de aparas que de recortes; não houve variação no volume de resíduos produzido ao longo das quatro semanas do mês.

\section{STATUS DA SUBMISSÃO}

Recebido: 10 nov., 2013

Aceito: 26 dez., 2016

\section{AUTOR(ES) PARA CORRESPONDÊNCIA}

\section{Cândida Pereira da Silva}

Departamento de Engenharia Florestal, Universidade Federal do Tocantins - UFT, Rua Badejós, Lote 7, Chácaras 69/72, Zona Rural, CEP 77402-970, Gurupi, TO, Brasil e-mail: engcandida@gmail.com

\section{REFERENCIAS}

Abreu LB, Mendes LM, Silva JRM, Paula LER, Mori FA. Avaliação de resíduos de painéis de madeira gerados por indústrias moveleiras para aproveitamento na confecção de pequenos objetos: estudo de caso. Ciência e Agrotecnologia 2009; 33: 1747-1751.

Abreu YV, Azevedo MRM. Racionamento de energia elétrica de 2001: o estado do Tocantins. Málaga: Universidade de Málaga; 2009. 105 p.

Fagundes HAV. Produção de madeira serrada e geração de resíduos do processamento de madeira de florestas plantadas no Rio Grande do Sul [dissertação]. Porto Alegre: Universidade Federal do Rio Grande do Sul; 2003.

Fontes PJP. Auto-suficiência energética em serraria de pinus e aproveitamento dos resíduos [dissertação]. Curitiba: Universidade Federal do Paraná; 1994.

Gomes JI, Sampaio SS. Aproveitamento de resíduos de madeira em três empresas madeireiras do estado do Pará. Belém: Embrapa Amazônia Oriental; 2004. 5 p. Comunicado Técnico n. 102.

Instituto Brasileiro de Desenvolvimento Floresta - IBDF. Madeiras da Amazônia: características e utilização. Brasília: Estação experimental de Curuá-Una; 1988. vol. 2. 
Kozak PA, Cortez AM, Schirmerc WN, Caldeira MVW, Balbinot R. Identificação, quantificação e classificação dos resíduos sólidos de uma fábrica de móveis. Revista Acadêmica: Ciências Agrárias e Ambientais 2008; v. 6, n. 2, p. 203-212.

Lima EG, Silva DA. Resíduos gerados em indústrias de móveis de madeira situadas no pólo moveleiro de Arapongas-PR. Revista Floresta 2005; 35(1): 105-116. http://dx.doi.org/10.5380/rf.v35i1.2434.

Lima JT, Silva JRM, Vieira RS. Aproveitamento de resíduos gerados no processamento da madeira de eucalipto. In: Oliveira JTS, Fiedler NC, Nogueira M. Tecnologias aplicadas ao setor madeireiro III. Jerônimo Monteiro: UFES; 2007. p. 255-290.

Lima JT, Vieira RS, Silva JRM, Pedreschi R. Uso da madeira de Eucalyptus selecionado para a confecção de móveis de alta qualidade. In: Anais do IX encontro brasileiro em madeiras e estruturas de madeiras [CD-ROM]; 2004; Cuiabá. Cuiabá: Universidade Federal do Mato Grasso; 2004. p. 1-14.
Mady FTM. Conhecendo a madeira: informações sobre 90 espécies comerciais. Manaus: Programa de Desenvolvimento Tecnológico, SEBRAE; 2000. 212 p.

Silas W. Gurupienses tem um "pé atrás" ao se tratar de empreendimentos [online]. Joinville: A Notícia; 2010. [citado em 2011 nov. 3]. Disponível em: http://www. anoticia to.com.br/noticias.php?IdNoticia $=12236$

Silva, J. R. M. Diagnóstico da indústria madeireira de Lavras/ MG: relatório técnico. Lavras: FAPEMIG, UFLA; 1999.

Vieira RS, Lima JT, Silva JRM, Hein PRG, Bailleres H, Baraúna EEP. Small wooden objects using eucalypt sawmill wood waste. BioResources 2010; 5: 1463-1472.

Vieira RS. Uso de madeira de Eucalyptus selecionado para confecção de móveis de alta qualidade [monografia]. Lavras: Universidade Federal de Lavras; 2003. 32 p.

Vieira RV. Pequenos Objetos de Madeira de Eucalipto: possibilidade de aproveitamento de resíduos [dissertação]. Lavras: Universidade Federal de Lavras; 2006. 94 p. 


\section{Errata}

No artigo “Quantificação de Resíduos Produzidos nas Indústrias Madeireiras de Gurupi, TO”, com número de DOI: http://dx.doi.org/10.1590/2179-8087.065613, publicado no periódico Floresta e Ambiente, vol. 24, e00065613, na primeira página:

Onde se lia:

Cândida Pereira da Silva ${ }^{1}$, Renato da Silva Vieira ${ }^{1}$, Iolanda Carvalho Silva ${ }^{1}$, Aline Silvestre Pereira ${ }^{1}$, Edy Eime Pereira Baraúna ${ }^{1}$

${ }^{1}$ Universidade Federal do Tocantins - UFT, Gurupi/TO, Brasil

Leia-se:

Cândida Pereira da Silva ${ }^{1}$, Renato da Silva Vieira ${ }^{2}$, Iolanda Carvalho Silva ${ }^{1}$, Aline Silvestre Pereira Dornelas ${ }^{1}$, Edy Eime Pereira Baraúna ${ }^{3}$

${ }^{1}$ Universidade Federal do Tocantins - UFT, Gurupi/TO, Brasil

${ }^{2}$ Departamento de Ciências Agrárias, Universidade Federal de São João Del Rei - UFSJ, Sete Lagoas/MG, Brasil

${ }^{3}$ Instituto de Ciências Agrárias - ICA, Universidade Federal de Minas Gerais - UFMG, Montes Claros/ MG, Brasil 